\title{
Time varying controllers in discrete-time decentralized control
}

\author{
C. Deliu ${ }^{1}$ \\ Anton A. Stoorvogel ${ }^{2}$ \\ Ali Saberi ${ }^{3}$ \\ Sandip Roy ${ }^{3}$ \\ Babak Malek $^{3}$
}

\begin{abstract}
In this paper, we consider the problem of finding a time-varying controller which can stabilize a decentralized discrete-time system. In continuous-time, it was already known that time-varying decentralized controllers can achieve stabilization in cases where time-invariant decentralized controllers cannnot. This paper works out the details for the discrete-time case.
\end{abstract}

\section{INTRODUCTION}

The result presented here contributes to our ongoing study of the stabilization of decentralized systems. The eventual goal of this study is the design of controllers for decentralized systems that achieve not only stabilization but also high performance. As a first step toward this design goal, we are currently looking for tight conditions on a decentralized plant for the existence of stabilizing controllers.

To motivate and introduce the main result in the article, let us briefly review foundational studies on both decentralized control and saturating control systems. We recall that a necessary and sufficient condition for stabilization of a decentralized system using linear, time-invariant state-space controllers is given in Wang and Davison's classical work [6]. They obtain that stabilization is possible if and only if all decentralized fixed modes of a plant are in the open lefthalf plane, and give specifications of and methods for finding these decentralized fixed modes. Numerous further characterizations of decentralized stabilization (and fixed modes) have been given, see for instance the work of Corfmat and Morse [2]. For time-varying controllers, the result for continuoustime systems goes back to the paper [1]. In this paper, it was established that there are systems for which there do not exist time-invariant, linear decentralized controllers while there do exist time-varying, linear decentralized controllers. Moreover, they obtain that stabilization is possible if and only if all quotient fixed modes of a plant are in the open lefthalf plane. For nonlinear, time-invariant systems some first results are presented in [5]. It has been shown in [4] that nonlinear, time-varying decentralized controllers can only stabilize a system when it can also be stabilized by a linear, time-varying decentralized controller.

\footnotetext{
${ }^{1}$ Department of Mathematics, and Computing Science, Eindhoven Univ. of Technology, P.O. Box 513, 5600 MB Eindhoven, The Netherlands. Department of Electrical Engineering, Mathematics and Computer Science, Delft Univ. of Technology, P.O. Box 5031, 2600 GA Delft, The Netherlands, E-mail: c.deliu@tue.nl

${ }^{2}$ Department of Electrical Engineering, Mathematics, and Computing Science, University of Twente, P.O. Box 217, 7500 AE Enschede, The Netherlands. E-mail: A.A.Stoorvogel@utwente.nl

${ }^{3}$ School of Electrical Engineering and Computer Science, Washington State University, Pullman, WA 99164-2752, U.S.A. E-mail: \{bmalek,sroy,saberi\}@eecs.wsu.edu.
}

In this paper we focus on time-varying, decentralized controllers for discrete-time systems. The continuous-time results in [1], [4] show that the existence results are related to the so-called quotient fixed modes while for time-invariant controllers the existence is related to decentralized fixed modes. Not surprisingly, in this case stabilization by timeinvariant controllers is possible if the decentralized fixed modes are in the open unit disc while for time-varying controllers stabilization is possible if the quotient fixed modes are in the open unit disc

In this paper, we first present our main results and then we present in Section III, a detailled outline of the proof of the crucial underlying lemma.

\section{MAIN RESULT}

Consider a decentralized control system $\Sigma$ :

$$
\Sigma:\left\{\begin{aligned}
x(k+1) & =A x(k)+\sum_{i=1}^{\nu} B_{i} u_{i}(k) \\
y_{i}(k) & =C_{i} x(k), \quad i=1, \ldots, \nu,
\end{aligned}\right.
$$

As in many control systems the first objective is to find a controller which can stabilize the given system under the presented decentralized structure. In other words, when do there exists $\nu$ controllers of the form:

$$
\Sigma_{i}:\left\{\begin{array}{l}
z_{i}(k+1)=K_{i} z_{i}(k)+L_{i} y_{i}(k), \quad z_{i} \in \mathbb{R}^{s_{i}} \\
u_{i}(k+1)=M_{i} z_{i}(k)+N_{i} y_{i}(k) .
\end{array}\right.
$$

such that the interconnection of (1) and (2) is asymptotically stable. From the paper [6] it is known for continuous-time systems that this is related to so-called decentralized fixed modes:

Definition 1 Consider the system $\Sigma . \lambda \in \mathbb{C}$ is called a decentralized fixed mode if for all matrices $H_{1}, \ldots H_{\nu}$ of appropriate dimension we have

$$
\operatorname{det}\left(\lambda I-A-\sum_{i=1}^{\nu} B_{i} H_{i} C_{i}\right)=0
$$

The following result goes back to [2], [6] for the continuous-time. The discrete-time equivalent follows quite directly and can for instance be found explicitly in [3]

Theorem 1 A necessary and sufficient condition for the existence of a decentralized feedback control law of the form (2) for the system $\Sigma$ such that the closed loop system is asymptotically stable is that all the fixed modes of the system are asymptotically stable (i.e. in the unit disc). 
In case the above condition is not satisfied a natural question is to check whether we can find a suitable controller if we expand the class of controllers to time-varying or nonlinear systems.

For time-varying controllers, again for continuous-time systems it is known that the result is related to the so-called quotient fixed modes. This result was initially presented in [1] while later further insight was obtained in the paper [4] . Let is first formally define quotient fixed modes:

Definition 2 Consider the system (1). We define a directed graph with nodes $\{1, \ldots, \nu\}$ with an edge from node $i$ to node $j$ if there exists an integer $k$ such that $C_{j} A^{k} B_{i} \neq 0$. Let $\nu^{*}$ be the number of strongly connected components of this directed graph. Let $u_{j}^{*}$ be the union of all inputs associated to the $j$ 'th strongly connected component, i.e. $u_{i}$ is part of $u_{j}^{*}$ if and only if node $i$ is part of the $j$ th strongly connected component of the graph. Similarly let $y_{j}^{*}$ be the union of all outputs associated to the $j$ 'th strongly connected component.

Without loss of generality we assume that the nodes are ordered in such a way that strongly connected component $j$ consists of nodes $\left\{i_{j-1}+1, \ldots, i_{j}\right\}$ with $i_{0}=0$ an $i_{\nu^{*}}=\nu$. Moreover, strongly connected component $j_{1}$ can connect to strongly connected component $j_{2}$ only if $j_{2} \leq j_{1}$.

Using the above, the matrices in the system equation will, in an appropriate basis have the following triangular structure

$$
\begin{aligned}
A & =\left(\begin{array}{cccc}
\tilde{A}_{1} & \times & \cdots & \times \\
0 & \tilde{A}_{2} & \ddots & \vdots \\
\vdots & \ddots & \ddots & \times \\
0 & \cdots & 0 & \tilde{A}_{\nu^{*}}
\end{array}\right), \\
B & =\left(\begin{array}{cccccc}
B_{1}^{*} & B_{2}^{*} & \cdots & B_{\nu^{*}}^{*}
\end{array}\right)=\left(\begin{array}{cccc}
\tilde{B}_{1} & \times & \cdots & \times \\
0 & \tilde{B}_{2} & \ddots & \vdots \\
\vdots & \ddots & \ddots & \times \\
0 & \cdots & 0 & \tilde{B}_{\nu^{*}}
\end{array}\right), \\
C & =\left(\begin{array}{c}
C_{1}^{*} \\
C_{2}^{*} \\
\vdots \\
C_{\nu^{*}}^{*}
\end{array}\right)=\left(\begin{array}{cccc}
\tilde{C}_{1} & \times & \cdots & \times \\
0 & \tilde{C}_{2} & \ddots & \vdots \\
\vdots & \ddots & \ddots & \times \\
0 & \cdots & 0 & \tilde{C}_{\nu^{*}}
\end{array}\right),
\end{aligned}
$$

where

$$
\begin{aligned}
B_{j}^{*} & =\left(\begin{array}{lll}
B_{i_{j-1}+1} & \cdots B_{i_{j}-1} & B_{i_{j}}
\end{array}\right) \\
C_{j}^{*} & =\left(\begin{array}{c}
C_{i_{j-1}+1} \\
\vdots \\
C_{i_{j}-1} \\
C_{i_{j}}
\end{array}\right)
\end{aligned}
$$

and

$$
\begin{aligned}
\tilde{B}_{j}^{*} & =\left(\begin{array}{lll}
\tilde{B}_{i_{j-1}+1} & \cdots \tilde{B}_{i_{j}-1} & \tilde{B}_{i_{j}}
\end{array}\right) \\
\tilde{C}_{j}^{*} & =\left(\begin{array}{c}
\tilde{C}_{i_{j-1}+1} \\
\vdots \\
\tilde{C}_{i_{j}-1} \\
\tilde{C}_{i_{j}}
\end{array}\right)
\end{aligned}
$$

for $j=1, \ldots, \nu^{*}$.

We call $\lambda \in \mathbb{C}$ a quotient fixed mode if $\lambda$ is a (centralized) uncontrollable or unobservable eigenvalue of $\left(\tilde{A}_{j}, \tilde{B}_{j}, \tilde{C}_{j}\right)$ for some $j \in\left\{1, \ldots, \nu^{*}\right\}$

It is not difficult to verify, using the upper-diagonal structure, that if we find stabilizing controllers for the subsystems:

$$
\Sigma^{j}:\left\{\begin{aligned}
x_{j}(k+1) & =\tilde{A}_{j} x(k)+\sum_{i=i_{j-1}+1}^{i_{j}} \tilde{B}_{i} u_{i}^{*}(k) \\
y_{i}(k) & =\tilde{C}_{i} x(k), \quad i=i_{j-1}, \ldots, i_{j},
\end{aligned}\right.
$$

for $j=1, \ldots, \nu^{*}$ then if we combine these controllers we find a stabilizing controller for the overall system. Conversely, we can only find a decentralized controller for the overall system if we can find decentralized controllers for each of the subsystems of the form (3). If all quotient fixed modes are inside the unit circle then these subsystems are all (centrally) stabilizable and detectable. Moreover, the graph associated with these subsystems is strongly connected.

The claim is that a decentralized system which is centrally stabilizable and detectable and whose associated graph is strongly connected can always be stabilized in a decentralized manner if we allow for time-varying controllers. This would immediately yield that the overall system is stabilizable by a decentralized time-varying controller if and only if all quotient fixed modes are inside the unit circle. The above claim which is crucial for this paper is formulated in the following lemma:

Lemma 1 Consider a system $\Sigma$ of the form (1) which is centrally stabilizable and detectable and which is such that the associated directed graph is strongly connected. Then there exist $\nu$ linear, time-varying controllers of the form (4) such that the resulting closed loop system is asymptotically stable.

The above lemma immediately yields the main result of this paper:

Theorem 2 Consider a system $\Sigma$ of the form (1) for which all quotient fixed modes are inside the unit circle. Then there exist $\nu^{*}$ linear, time-varying controllers of the form:

$$
\Sigma_{i}:\left\{\begin{array}{l}
z_{i}(k+1)=K_{i}(k) z_{i}(k)+L_{i}(k) y_{i}(k), \quad z_{i} \in \mathbb{R}^{s_{i}} \\
u_{i}(k+1)=M_{i}(k) z_{i}(k)+N_{i}(k) y_{i}(k) .
\end{array}\right.
$$

such that the resulting closed loop system is asymptotically stable. 
It is easily verified that quotient fixed modes are a subset of the decentralized fixed modes which makes the above theorem consistent with Theorem 1. However, there are examples of systems which can be stabilized via decentralized time-varying controllers but which can not be stabilized via decentralized time-invariant controllers.

\section{PRoOF OF LEMMA 1}

Consider a system $\Sigma$ of the form (1) which is centrally stabilizable and detectable and which is such that the associated directed graph is strongly connected. We will first prove there exists a static decentralized and time-varying (but periodic) preliminary feedback such that the system is uniformly detectable from channel 1 . Let $x$ be such that

$$
\left(\begin{array}{c}
C_{1} \\
C_{1} A \\
\vdots \\
C_{1} A^{n-1}
\end{array}\right) x=0
$$

Next, consider an initial condition $x(0)=x$ such that $y_{1}(j)=0$ for $j=0, \ldots, n-1$. Assume $x(n) \neq 0$ and $x(n)$ is observable through another channel $j$, i.e. $C_{j} A^{s} x(n) \neq 0$ for some $s \geq 0$. Note that this implies $C_{j} A^{p} x \neq 0$ for $p=s+n$. We will prove that there exists a preliminary feedback such that $y_{1}(\tilde{p}) \neq 0$ for some $\tilde{p}>n$.

Consider the associated strongly connected directed graph. Let $\left\{j, j_{v}, \ldots, j_{1}, 1\right\}$ be the shortest path from node $j$ to node 1. By definition, this implies there exists $p_{1}, \ldots, p_{v+1}$ such that:

$$
\begin{aligned}
& C_{1} A^{p_{1}} B_{j_{1}} \neq 0 \quad C_{j_{1}} A^{p_{2}} B_{j_{2}} \neq 0 \quad \cdots \\
& C_{j_{v-1}} A^{p_{v}} B_{j_{v}} \neq 0 \quad C_{j_{v}} A^{p_{v+1}} B_{j} \neq 0
\end{aligned}
$$

Then there exists $K_{1}, \ldots, K_{v+1}$ such that

$$
\begin{aligned}
& C_{1} A^{p_{1}} B_{j_{1}} K_{1} C_{j_{1}} A^{p_{2}} B_{j_{2}} K_{2} \times \cdots \\
& \quad \times C_{j_{v-1}} A^{p_{v}} B_{j_{v}} K_{v} C_{j_{v}} A^{p_{v+1}} B_{j} K_{v+1} C_{j} A^{p} x \neq 0
\end{aligned}
$$

From the fact that we have chosen a shortest path it is easily verified that

$$
\begin{aligned}
C_{j_{\ell-1}} A^{p_{\ell}} B_{j_{\ell}} & K_{j_{\ell}} C_{j_{\ell}} A^{p_{\ell+1}} B_{j_{\ell+1}} \\
& =C_{j_{\ell-1}} A^{p_{\ell}}\left(A+B_{j_{\ell}} K_{j_{\ell}} C_{j_{\ell}}\right) A^{p_{\ell+1}} B_{j_{\ell+1}}
\end{aligned}
$$

for any $\ell \in\{1, \ldots, v\}$ (with $j_{0}=1$ ). If this would not be true then

$$
C_{j_{\ell-1}} A^{p_{\ell}} A A^{p_{\ell+1}} B_{j_{\ell+1}} \neq 0
$$

which would imply an edge from $j_{\ell+1}$ to $j_{\ell-1}$ and a shorter path from $j$ to 1 . Choose the preliminary feedback:

$$
\begin{gathered}
u_{j}(p)=\varepsilon K_{v+1} y_{j}(p)+v_{j}(p) \\
u_{j_{v}}\left(p+p_{v}+1\right)=\varepsilon K_{v} y_{j_{v}}\left(p+p_{v}+1\right) \\
+v_{j_{v}}\left(p+p_{v}+1\right) \\
u_{j_{v-1}}\left(p+p_{v}+p_{v-1}+2\right)= \\
\varepsilon K_{v-1} y_{j_{v-1}}\left(p+p_{v}+p_{v-1}+2\right) \\
+v_{j_{v-1}}\left(p+p_{v}+p_{v-1}+2\right) \\
\vdots \quad \\
u_{j_{1}\left(p+p_{v}+\cdots+p_{1}+v\right)}= \\
\varepsilon K_{1} y_{j_{1}}\left(p+p_{v}+\cdots+p_{1}+v\right) \\
+v_{j_{1}}\left(p+p_{v}+\cdots+p_{1}+v\right)
\end{gathered}
$$

while for all other channels and all other time instances we have no preliminary feedback. After this preliminary feedback we obtain a system:

$$
\Sigma:\left\{\begin{aligned}
x(k+1) & =A_{\varepsilon}(k) x(k)+\sum_{i=1}^{\nu} B_{i} v_{i}(k) \\
y_{i}(k) & =C_{i} x(k), \quad i=1, \ldots, \nu,
\end{aligned}\right.
$$

for $k=1, \ldots, \tilde{p}$ where

$$
\tilde{p}=p+p_{v}+\ldots+p_{1}+v
$$

In that case $x(0)=x$ yields

$$
y_{1}(\tilde{p}) \neq 0
$$

for all $\varepsilon>0$ given $v_{i}(k)=0$ for $k=1, \ldots, \tilde{p}$ and $i=1, \ldots, \nu$. Next, consider an initial condition $\tilde{x}$ such that $y_{1}(k)=0$ for $k=1, \ldots, \tilde{p}$ while there exists a channel $\tilde{j}$ such that $C_{\tilde{j}} A^{s} \tilde{x} \neq 0$ for some $s>\tilde{p}$. This clearly implies that

$$
A^{\tilde{p}} \tilde{x} \neq 0
$$

but then

$$
\bar{x}_{\varepsilon}=\prod_{k=1}^{\tilde{p}} A_{\varepsilon}(k) \tilde{x} \rightarrow A^{\tilde{p}} \tilde{x}
$$

as $\varepsilon \rightarrow 0$ and hence for $\varepsilon$ small enough we have

$$
\bar{x}_{\varepsilon} \neq 0 \quad C_{\tilde{j}} A^{s-\tilde{p}} \bar{x}_{\varepsilon} \neq 0
$$

Then, as before, we can find a preliminary feedback for $k=$ $\tilde{p}, \ldots, \bar{p}$ such that we obtain a system (5) with the property that for $x(0)=\tilde{x}$ we obtain:

$$
y_{1}(\bar{p}) \neq 0
$$

given $v_{i}(k)=0$ for $k=1, \ldots, \bar{p}$ and $i=1, \ldots, \nu$. We can repeat this algorithm again if we can find an $\hat{x}$ such that $y_{1}(k)=0$ for $k=1, \ldots, \bar{p}$ given $v_{i}(k)=0$ for $k=1, \ldots, \bar{p}$ and $i=1, \ldots, \nu$ while

$$
C_{j} A^{k} \hat{x} \neq 0
$$

for some $k>\bar{p}$. However, because of the linearity of the system, the algorithm will end after at most $n$ steps because of the dimension of the state space. After the algorithm is completed we find that there exists $p>0$ such that for any 
initial condition $x_{0}$ either it is observable through $y_{1}$ on the time interval $[0, p]$ or

$$
C_{j} A^{s} x_{0}
$$

is equal to zero for all $j=1, \ldots, \nu$ and for all $s>p$. This implies that $A^{p} x_{0}$ is (centrally) unobservable for the original system, i.e.

$$
x_{0} \in \operatorname{ker} A^{p}+\langle\operatorname{ker} C, A\rangle
$$

where

$$
C=\left(\begin{array}{c}
C_{1} \\
C_{2} \\
\vdots \\
C_{\nu}
\end{array}\right)
$$

For a proof of (6), see Appendix. It is then easily verified that $x(k)=A^{k} x_{0}$ given $v_{i}(k)=0$ for $k=1, \ldots, \bar{p}$ and $i=1, \ldots, \nu$. Since, by assumption $(C, A)$ was detectable we find that $A^{k} x_{0} \rightarrow 0$ as $k \rightarrow \infty$. If we extend this preliminary feedback periodically it is easily verified that we obtain a system which is uniformly detectable through channel 1 .

However, we also need stabilizability. We have chosen a preliminary feedback for $k=1, \ldots, p$ to achieve detectability. Next, we can, through a dual argument, choose a preliminary feedback for $k=p+1, \ldots, q$ such that the system becomes stabilizable. Clearly, this need not be done succesively in time but it makes the argument a lot easier. We simply look at $x(p)$ and check whether we can make those states stabilizable through channel 1 on the interval $[p+1, q]$. This argument is completely dual to the design to make the system detectable through channel 1 on the interval $[0, p]$.

In this way, we obtain a preliminary feedback such that we have detectability on the interval $[0, p]$ and stabilizability on the interval $[p+1, q]$. By making this preliminary feedback periodic, we obtain a system which is uniformly stabilizable and detectable through channel 1 . It is well known that this implies that there exists a stabilizing, dynamic controller for channel 1. The latter completes the proof of Theorem 1.

\section{CONClusion}

This paper presents a necessary and sufficient conditions for the existence of stabilizing time-varying linear controllers for a discrete-time decentralized system. Like the earlier continuous-time results, the proof of the existence of a suitable controller is constructive but is certainly not efficient enough to generate an easily implementable controller. For decentralized system, there is still a strong need for suitable design methodologies which is a main topic of our future research. The lack of a suitable design methodology for general decentralized systems is not limited to time-varying designs. Also if all decentralized fixed modes are asymptotically stable, then we still do not have a suitable design for a general decentralized system. For certain systems with special additional structure such designs of course exist.

\section{APPENDIX}

Lemma 2 Consider the matrices $A \in \mathbb{R}^{n \times n}, C \in \mathbb{R}^{p \times n}$ and the subspace

$$
W=\left\{x \in \mathbb{R}^{n} ; C A^{k} x=0, \forall k \geq s\right\},
$$

where $s \geq n$. Then we have

$$
W=\operatorname{ker} A^{s}+\langle\operatorname{ker} C, A\rangle
$$

Proof.

$x \in \operatorname{ker} A^{s} \Rightarrow A^{s} x=0 \Rightarrow C A^{k} x=0, \forall k \geq s \Rightarrow x \in W_{s}$,

So

$$
\operatorname{ker} A^{s} \subset W_{s}
$$

$$
x \in\langle\operatorname{ker} C, A\rangle \Rightarrow C A^{k} x=0, \forall k \in \mathbb{N} \Rightarrow x \in W_{s}
$$

which implies

$$
\langle\operatorname{ker} C, A\rangle \subset W_{s}
$$

From (7) and (8) we find

$$
\langle\operatorname{ker} C, A\rangle+\operatorname{ker} A^{s} \subset W_{s}
$$

Consider now $x \in W_{s}$. In an appropriate basis we can write

$$
A=\left(\begin{array}{cc}
A_{11} & A_{12} \\
0 & A_{22}
\end{array}\right), C=\left(\begin{array}{ll}
0 & C_{2}
\end{array}\right)
$$

with $A_{11} \in \mathbb{R}^{n_{1} \times n_{1}}, A_{22} \in \mathbb{R}^{n_{2} \times n_{2}}$ and $\left(C_{2}, A_{22}\right)$ observable. In the same basis we can write $x=\left(\begin{array}{cc}x_{1} & x_{2}\end{array}\right)^{T}$ and since $C A^{k} x=0 \forall k \geq s$, we find $C_{2} A_{22}^{k} x_{2}=0, \forall k \geq s$. This implies $A_{22}^{s} x_{2} \in\left\langle\operatorname{ker} C_{2}, A_{22}\right\rangle$, so $A_{22}^{s} x_{2}=0$. Since $s \geq n_{2}$ (the size of $A_{22}$ ), we find that

$$
x_{2} \in \operatorname{ker} A_{22}^{s}=\operatorname{ker} A_{22}^{k}, \forall k \geq n_{2}
$$

Let us write now

$$
\left(\begin{array}{l}
x_{1} \\
x_{2}
\end{array}\right)=\left(\begin{array}{l}
\tilde{x}_{1} \\
x_{2}
\end{array}\right)+\left(\begin{array}{c}
x_{1}-\tilde{x}_{1} \\
0
\end{array}\right)
$$

Obviously the second vector from this decomposition is in $\langle\operatorname{ker} C, A\rangle$, so we need to find $\tilde{x}_{1}$ such that the first vector is in $\operatorname{ker} A^{s}$, or equivalently ( since $A_{22}^{s} x_{2}=0$ ),

$$
A_{11}^{s} \tilde{x}_{1}+\sum_{i=0}^{s-1} A_{11}^{s-i-1} A_{12} A_{22}^{i} x_{2}=0
$$

Using (10), the previous equation becomes

$$
A_{11}^{s} \tilde{x}_{1}+\sum_{i=0}^{n_{2}-1} A_{11}^{s-i-1} A_{12} A_{22}^{i} x_{2}=0
$$

We have that $\sum_{i=0}^{n_{2}-1} A_{11}^{s-i-1} A_{12} A_{22}^{i} x_{2} \in \operatorname{im} A_{11}^{s-n_{2}}=$ $\operatorname{im} A_{11}^{n_{1}}$ (because $s-n_{2} \geq n_{1}$ ). So there exists $\hat{x}$ such that $A_{11}^{s} \hat{x}=\sum_{i=0}^{n_{2}-1} A_{11}^{s-i-1} A_{12} A_{22}^{i} x_{2}$ since $\operatorname{im} A_{11}^{s}=\operatorname{im} A_{11}^{n_{1}}$ $\left(s \geq n_{1}\right)$. Choosing now $\tilde{x}=-\hat{x}$, (11) is satisfied. 


\section{REFERENCES}

[1] B.D.O. ANDERSON AND J.B. Moore, "Time-varying feedback laws for decentralized control", IEEE Trans. Aut. Contr., 26(5), 1981, pp. 1133-1139.

[2] J.P. Corfmat And A.S. Morse, "Decentralized control of linear multivariable systems", Automatica, 12(5), 1976, pp. 479-495.

[3] C. Deliu, B. Malek, S. Roy, A. SABeri, AND A.A. Stoorvogel, "Decentralized control with input saturation", Submitted for publication, 2008.

[4] Z. Gong AND M. AldeEn, "Stabilization of decentralized control systems", J. Math. Syst. Estim. Control, 7(1), 1997, pp. 1-16.

[5] A.A. Stoorvogel, J. Minteer, and C. Deliu, "Decentralized control with saturation: a first step toward design", in American Control Conference, Portland, OR, 2005, pp. 2082-2087.

[6] S.H. WANG AND E.J.DAVISON, "On the stabilization of decentralized control systems", IEEE Trans. Aut. Contr., 18(5), 1973, pp. 473-478. 